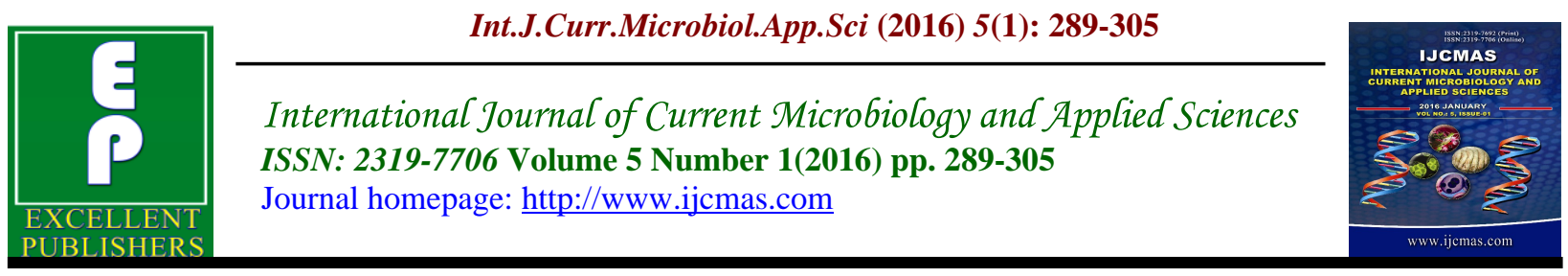

Original Research Article

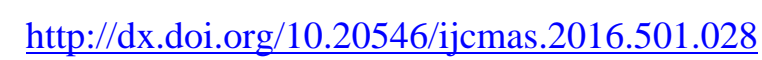

\title{
Sputum Bacteriology in Patients with Acute Exacerbation of Chronic Obstructive Pulmonary Disease
}

\author{
Dalia Saad ElFeky ${ }^{1 *}$, Heba Mohammed Elmandory ${ }^{2}$, \\ Mohamed Galal ${ }^{3}$ and Mohamed Abdel Hakim ${ }^{3}$ \\ ${ }^{1}$ Department of Medical Microbiology and Immunology, Faculty of Medicine, \\ Cairo University, Egypt \\ ${ }^{2}$ Department of Chest Diseases, Police Hospital, Agouza, Egypt \\ ${ }^{3}$ Department of Chest Diseases and Tuberculosis, Faculty of Medicine, Cairo University, Egypt \\ *Corresponding author
}

\section{A B S T R A C T}

Keywords

COPD, Acute exacerbation, sputum specimens, Wash technique, Quantitative culture, Streptococcus pneumoniae

Article Info

Accepted: 14 December 2015 Available Online: 10 January 2016
The present study was conducted to detect the most common bacterial pathogens associated with acute exacerbation of chronic obstructive pulmonary disease (AECOPD) and their antibiogram in Egyptian patients and to assess the quantitative culture technique of washed sputum specimens. Thirty patients diagnosed as AECOPD by pulmonary functions tests and clinical findings were subjected to physical examination, chest $\mathrm{x}$-ray, routine laboratory tests, as well as direct and quantitative culture of washed sputum specimens. Potential bacterial pathogens above the cutoff count were isolated in $66.6 \%$ of studied patients. The most common isolated bacterial pathogen was Streptococcus pneumoniae (43.3\%) followed by methicillin-resistant Staphylococcus aureus (MRSA) (10\%), Haemophilus influenzae (6.7\%) and finally Acinetobacter spp and Moraxella catarrhalis (3.3\% for each). Organisms were mostly sensitive to quinolones followed by ceftriaxone. Neither leucocytosis nor CRP was a good predictor of bacterial infections. There was a good correlation between gram-stained smear results and culture results. The wash technique improved the sputum smear and culture results. Bacterial infections play an important role in AECOPD. Quinolones are good therapeutic options. The wash technique could be used to improve the diagnostic value of sputum specimens.

\section{Introduction}

An exacerbation of chronic obstructive pulmonary disease (AECOPD) is defined as an event in the natural course of the disease characterized by a change in the patient's Baseline dyspnea, cough andlor sputum that is beyond normal day-to-day variations, acute in onset, and may warrant a change in regular medications in patient with underlying COPD (Rodriguez-Roisin, 2000). The most common causes of exacerbations are infection of the tracheobronchial tree and air pollution, but 
the cause of about one-third of severe exacerbations cannot be identified (GOLD, 2010; NICE, 2010).

The role of bacterial infection is controversial, but recent investigations have shown that at least $50 \%$ of patients have bacteria in high concentrations in their lower airways during exacerbations (Sethi et al., 2002). Haemophilus influenzae (H. influenzae), Streptococcus pneumoniae (S. pneumoniae), and Moraxella catarrhalis (M. catarrhalis) are the most common bacterial pathogens involved in an exacerbation (Sethi and Murphy, 2008).

Expectorated sputum is the most commonly used sample for diagnosis of lower respiratory tract infections (LRTI), which can be obtained easily and non-invasively (Ziyade and Yagci, 2010). However, sputum may show a heavy growth of commensal organisms because of the necessity to traverse a heavily contaminated oropharynx which may prevent the determination of the true epidemiologic agent. Using a wash technique and quantitative culture of sputum has been shown to decrease the number of contaminants by 100 to 1000 fold and has enhanced the value of sputum samples (Buenviaje, 1989; Ziyade and yagci, 2010).

This study was conducted to detect the most common bacterial pathogens associated with AECOPD and their antibiogram in Egyptian patients and to assess the quantitative culture technique of washed sputum specimens.

\section{Materials and Methods}

The study has been approved by the Research and Ethical Clearance Committee at the Department of Medical Microbiology and Immunology, Faculty of Medicine, Cairo University, as well as the local institutional review board of Giza Chest
Hospital. The study included 30 patients with AECOPD; 26 selected from outpatient clinic and 4 from inpatients in Giza-Chest Hospital during the period from September 2011 to October 2013. Patients were diagnosed as having COPD by evidenced of a ratio of post bronchodilator forced expiratory volume in first second (FEV1) to forced vital capacity (FVC) of less than $70 \%$ (NICE, 2010) and as having acute exacerbation by the presence of at least two of the following findings; worsening of dyspnea, increase sputum volume and increase sputum purulence (Anthonisen et al., 1987; GOLD, 2010). Patients with bronchial asthma, cystic fibrosis, broncheictasis or clinical radiological evidence of pneumonia as well as those who received antibiotic treatment within one week prior to collection of sputum samples were excluded from the study.

After giving an informed consent, all patients were subjected to history taking, general and local examination, chest $\mathrm{x}$-ray to exclude other diseases as pneumonia and pneumothorax, pulmonary function tests done by spirometery (American MedGraphics equipment, model no.762026101) after patients' confirmation that they did not receive bronchodilators and repeated 15 minutes after receiving salbutamol inhalation, in addition to measurement of arterial blood gases. Routine laboratory investigation including complete blood count (CBC) test and qualitative detection of C-reactive protein (CRP) by latex agglutination test as well as microbiological examination of sputum specimens were done for all patients.

\section{Pulmonary Function Tests}

These included; FVC, $\mathrm{FEV}_{1}, \mathrm{FEV}_{1}$ / FVC ratio, forced expiratory flow at 25\% (FEF $25 \%$ ), FEF at $50 \%$ (FEF $50 \%$ ), FEF at $75 \%$ 
(FEF 75\%), maximum EF (FEF Max), FEF at $25 \%-75 \%$ (FEF 25\% - 75\%), forced inspiratory flow at 50\% (FIF 50\%) and FEF $50 \%$ / FIF 50\%. All tests were done for 3 times and the best measurement was selected. The FVC and FEV1 values in these three curves should vary by no more than $5 \%$ or $100 \mathrm{ml}$, whichever was greater. The FEV1/FVC was taken from the technically acceptable curve with the largest sum of FVC and FEV1. Spirometry measurements were evaluated by comparison of the results with appropriate reference values based on age, height, sex, and race (Morris et al., 1971). The presence of a post bronchodilator FEV1/FVC $<70 \%$ confirms the presence of airflow limitation. Also all the previous tests were repeated 15 minutes after receiving $400 \mu \mathrm{g}$ of Salbutamol by inhalation and reversibility was estimated as regard FEV1 after change. Patients were classified into 4 Groups based on Post-Bronchodilator $\mathrm{FEV}_{1}$ (GOLD, 2010) as follow; Mild; $\mathrm{FEV}_{1} \geq 80 \%$ predicted, Moderate; $50 \% \leq \mathrm{FEV}_{1}<80 \%$ predicted, Severe; $30 \% \leq \mathrm{FEV}_{1}<50 \%$ predicted and Very Severe; $\mathrm{FEV}_{1}<30 \%$ predicted.

\section{Microbiological Examination of Sputum Specimens}

\section{Specimen Collection}

Sputum samples were collected from all participants according to standard guidelines (Baron and Thomson, 2012). Briefly, sputum samples were collected with patients in the sitting position and after washing their mouth. Patients were instructed to take a deep breath, hold it for a moment and then cough vigorously into $100 \mathrm{ml}$ sterile universal containers. Specimens were transported and processed within 2 hours in the laboratory of the Department of Medical Microbiology and Immunology, Faculty of Medicine, Cairo University.

\section{Macroscopic and Microscopic Examination}

The specimens were subjected to macroscopic examination for physical appearance of the specimens and screening by microscopic examination of gram-stained smears to determine the acceptability of the specimen for culture. The area of maximal purulence was examined for polymorph leukocytes (PMNL) and squamous epithelial cells (SEC). Sputum specimens containing over 10 SEC / low power field (LPF) were rejected (American Thoracic Society, 1995; Baron and Thomson, 2012), while accepted samples were divided into three groups by means of Geckler criteria (Geckler et al., 1977; Roche et al., 2007); i) < 10 SEC and > 25 leukocyte /LPF, ii) < 10 SEC and $10-25$ leukocyte/LPF, iii) $<10$ SEC and $<10$ leukocyte/LPF

\section{Wash Technique}

The accepted specimens were subjected to a wash technique to decrease contaminants as described in earlier studies (Bartlett and Finegold, 1978; Cao et al., 2004; Ziyade and Yagci, 2010). The specimens were mixed with physiological saline (1:10 vol), vortexed and centrifuged for 10 minutes at $1500 \mathrm{rpm}$. An equal volume of N- acetyl- L cysteine was added to the pellet for homogenization and the mixture was incubated at $37^{\circ} \mathrm{C}$ for 15 minutes.

\section{Processing of Washed Sputum Specimens}

Gram stained smears were prepared from washed specimens and screened for the predominant bacteria. Washed sputum samples were also inoculated into sheep blood agar, Chocolate agar and MacConkey agar plates. Two techniques were used for inoculation of the culture media; $i$. Semiquantitatively using streak plate 
method, ii. Quantitatively using serial dilutions of specimens followed by inoculation of $10 \mu 1$ of $10^{-1}$ and $10^{-2}$ dilutions onto culture media (Allegra et al., 2005; Roche et al., 2007). The plates were then incubated at $37 \mathrm{C}$ at $5-10 \% \mathrm{CO} 2$ for $24-48$ hours. Bacterial growth in agar plates was identified by the standard microbiological methods. An arbitrary cutoff point was chosen to minimize risk of dealing with contaminant. A potentially-pathogenic microorganism (PPM) had to grow in significant counts to be considered a potential causative agent of an exacerbation (Miravitles et al., 1999; Allegra et al., 2005). Isolated micro-organisms were regarded as significant and accepted as causative pathogens only if they reached a count of $10^{6} \mathrm{CFU} / \mathrm{mL}$, except for $S$. pneumoniae where $10^{5} \mathrm{CFU} / \mathrm{mL}$ was estimated to be sufficient (Miravitles et al., 1999; Allegra et al., 2005). The isolated micro-organisms were tested for their antimicrobial susceptibility pattern using disc diffusion method according to CLSI guidelines (CLSI, 2013).

\section{Statistical Analysis}

Data were statistically described in terms of mean \pm standard deviation ( \pm SD), and range, or frequencies (number of cases) and percentages when appropriate. Comparison of between the study groups was done using Chi square $(\chi 2)$ test. Exact test was used instead when the expected frequency is less than 5. Correlation between various variables was done using Pearson moment correlation equation for linear relation in normally distributed variables and Spearman rank correlation equation for non-normal variables. Accuracy was represented using the terms sensitivity, specificity, positive predictive value (PPV), negative predictive value (NPV), overall accuracy. $P$ value less than 0.05 was considered statistically significant. All statistical calculations were done using computer program SPSS (Statistical Package for the Social Science; SPSS Inc., Chicago, IL, USA release 15 for Microsoft Windows 2006).

\section{Results and Discussion}

This study included 30 patients with AECOPD selected from a total of 44 patients after exclusion of 4 patients due to inappropriate sputum specimens and 10 patients due to normal spirometery measurements.

\section{Sputum Culture and Antibiotic Susceptibility Testing}

Quantitative cultures of washed sputum specimens (figure 1) grew PPM above the cutoff count in 20 cases $(66.6 \%)$. Grampositive organisms represented the majority of the isolates (80\%), while Gram-negative organisms represent the remaining $20 \%$. The most prevalent isolated bacteria (table 1) were $S$. pneumoniae (13 cases; 43.3\%), followed by methicillin-resistant $S$. aureus (MRSA) (3 cases; 10\%), then $H$. influenzae ( 2 cases; $6.7 \%$ ), and lastly $M$. catarrhalis and Acinobacter spp. (1 case for each; $3.3 \%$ ). All the isolates were recovered from outpatients except the Acinetobacter and one MRSA isolates which were recovered from 2 hospitalized patients. Although all the isolates were detected by both the direct and quantitative methods, however, 3 false positive cases were detected by the direct method where they were positive by the direct method, however colony count for these cases was below the threshold cutoff value.

Ciprofloxacin and ceftriaoxone were effective against most of the bacterial isolates in this study, while increase resistance to erythromycin and penicillin. The most effective antimicrobial agents 
against $S$. pneumoniae isolates were ciprofloxacin and vancomycin (92.30\%) followed by amoxycillin-clavulinic acid, azythromycin and ceftriaxone $(76.92 \%$ for each), erythromycin (69.23\%) and finally penicillin (46.15\%). All S. aureus isolates were MRSA. The most effective antimicrobial agents against MRSA isolates was gentamycin, $(66.7 \%)$ followed by erythromycin and cirprofloxacin (33.3\% for each). The most effective antimicrobial agents to $H$. influenzae were ciprofloxacin and ceftriaxone while amoxycillin-clavulinic acid was ineffective. The $M$. cataralis isolate was sensitive to ciprofloxacin, amoxycillin-clavulinic acid and ceftriaxone while erythromycin was ineffective. The Acinetobacter isolate was sensitive to ciprofloxacillin \& amoxicillin-clavulinic acid, while ceftriaxone $\&$ erythromycin were ineffective

\section{Demographic and Clinical Data of the Patients: (Table 2)}

The majority of the patients $(93.3 \% ; 28$ patients) were males while only 2 patients $(6.7 \%)$ were females. The patients' age ranged from 40 to 82 years with a mean of $58.20 \pm 10.79$ years. Most patients were in the age group from 60 to 70 years $(36.7 \%)$. Age group 50-60 years old had the highest culture positivity (100\%; 8 patients). Although, $S$. pneumoniae was the most common isolated pathogen in all age groups, they were most predominant in the age group $60-70$ years old $(85.7 \% ; 6$ isolates) . Most of the patients were smokers $(76.6 \%)$. Body Mass Index (BMI) of patients ranged from 15.1 to 37.6 with a mean of $24.32 \pm$ $6.07 \mathrm{~kg} / \mathrm{m}^{2}$. Most patients were from urban areas $(83.3 \%)$. S. pneumoniae represent $75 \%$ and $62.5 \%$ of the isolates in rural and urban areas, respectively. However, MRSA, $H$. influenzae and Acinetobacter spp. were isolated only from patients from urban areas, while the only $M$. catarrhalis strain was isolated from a patient from rural areas. There were no statistical significant differences between different age groups, BMI groups, smokers and ex-smokers or rural and urban residency regarding culture results $(p$ value $=0.07,0.26,1.00$ and 0.64 respectively) or different types of isolated bacteria $(p$ value $=0.79,0.86,0.31$ and 0.23 respectively).

Post bronchodilator FEV1\% predicted ranged from 21 to $90 \%$ with a mean of 44.87 $\pm 19.08 \%$. The COPD patients were subdivided into subgroups according to FEV1\% of predicted as described earlier (table 2). There was no statistical significant difference between COPD subgroups as regards culture results $(\mathrm{p}$ value $=0.55)$ or the different types of isolated bacteria ( $p$ value $=0.53$ ) (table 3). Oxygen saturation in patients' blood (SO2 \%) ranged from 87 to $97 \%$ with a mean of $94.23 \pm 2.25 \%$. There was no statistical significant difference between hypoxic and non hypoxic groups regarding culture result ( $p$ value $=0.53$ ) but there was a statistical significant difference between hypoxic groups regarding different types of isolated bacteria ( $p$ value $=0.28$ ). All cases with $S$. pneumonia had no hypoxia, while the 3 cases with hypoxia had positive cultures for MRSA, $H$. influenzae and Acinetobacter spp. Partial pressure of oxygen in arterial blood (PO2) ranged from 53 to $99 \mathrm{mmHg}$ with a mean of $73.23 \pm 9.78 \mathrm{mmHg}$. There were no statistical significant difference between hypoxemic patients and non hypoxemic patients, regarding culture result ( $p$ value $=$ 0.66 ) or isolated bacteria ( $p$ value $=0.86$ ). Partial pressure of carbon dioxide in arterial blood (PCO2) ranged from 33 to $51 \mathrm{mmHg}$ with a mean of $39.87 \pm 5.15 \mathrm{mmHg}$. Only 3 patients $(10 \%)$ had hypercapnia. There were a statistical significant direct correlations of FEV1 \% predicted with $\mathrm{SO} 2$ 
$(p$ value $=0.001)$ and $\mathrm{PaO} 2$ ( $p$ value $=$ 0.001), while a statistical significant inverse correlation was present with $\mathrm{PaCO} 2$ ( $p$ value $=0.034)$.

Laboratory Data of the Patients: (Table 2)

Haematocrit (HCT) \% ranged from 34.9 to $51.1 \%$ with a mean of $42.86 \pm 3.86 \%$. Platelets count ranged from 150 to $551 \times 10^{3} /$ cmm with a mean of $258.40 \pm 97.23 \times 10^{3} /$ $\mathrm{cmm}$. Leucocytes count ranged from 4.4 to13.3 $\times 10^{3} / \mathrm{cmm}$ with a mean of $8.38 \pm$ $2.43 \times 10^{3} / \mathrm{cmm}$. All patients with leukocytosis (8 patients, $100 \%$ ) in addition to 12 patients, $(54.5 \%)$ out of 22 patients with normal leukocyte count (TLC) in CBC, had positive culture results. There was a statistical significant difference between high TLC group and normal TLC group regarding culture results ( $p$ value $=0.02$ ) but not as regards type of isolated bacteria ( $p$ value $=0.22)$. High TLC count had sensitivity, specificity, PPV and NPV of $40 \%, 100 \%, 100 \%$, and $45 \%$, respectively for predicting positivity of the culture, however, high TLC count was more predictive of $S$. aureus and $H$. influenzae rather than $S$. pneumoniae infections. Out of the 13 patients positive for $S$. pneumoniae, only 4 patients had high TLC while 2 patients out of the 3 patients with $S$. aurues and all patients positive for $H$. influenzae (2 patients) had high TLC. The positive cases with $M$. catarrhalis and Acinetobacter spp. (one case each) had normal TLC.

There was no statistically significant difference between patients with positive or negative $\mathrm{C}$-reactive protein (CRP) as regards culture positivity $(p$-value $=0.09)$. Positive CRP had sensitivity, specificity, PPV and NPP of $95 \%, 30 \% 73 \%$ and $75 \%$, respectively for prediction of culture positivity. The only case with negative CRP and positive culture grew $S$. pneumonia, while patients with positive CRP, their culture were positive for $S$. pneumoniae (12 isolates, 63.2\%), S. aureus (3 isolates, 15.8 $\%$ ), H. influenzae (2 isolates, 10.5\%), M. catarrhalis and Acinetobacter (1 isolate, $5.3 \%$ each). There was no statistical significant correlations between culture results and clinical and laboratory data except for TLC (a statistical significant direct correlation; $p$ value $=0.013$ ) and HCT (a statistical significant inverse correlation; $p$ value $=0.006)($ table 4$)$

\section{Macroscopic and Microscopic Examination of Sputum Specimens: (Table 5)}

Among positive culture cases, 12 patients $(60 \%)$ had purulent sputum, 7 patients (35\%) had mucopurulent sputum and 1 patient $(5 \%)$ had mucoid sputum, with a statistically significant difference among different physical characters of sputum as regards culture results $(p$ value $=0.019)$. Sensitivity, specificity, PPV and NPV of purulent and mucopurulent sputum for prediction of culture positivity were $95 \%$, $30 \%, 73 \%$ and $75 \%$, respectively. There was no statistical significant difference among different physical characters of sputum as regards the isolated bacteria ( $\mathrm{p}$ value $=$ 0.35). Most $S$. pneumoniae strains (9 isolates, $69.2 \%$ ) were isolated from purulent sputum; the 3 MRSA strains were isolated from the 3 types of sputum ( 1 isolate from each type). The 2 strains of $H$. influenzae were isolated from purulent and mucopurulent sputum (1 isolate in each type). The only $M$. catarralis strain was isolated from purulent sputum; while the Acenitobacter strain was isolated from mucopurulent sputum (1 isolate, 100\%).

There was no statistical significant difference between culture-positive and culture-negative cases regarding the 
different count of PMNL/LPF in sputum smears (table 5). The presence of PMNLs in Gram stained sputum smears had sensitivity, specificity, PPV, and NPV of $50 \%, 70 \%$, $76.9 \%$ and $41 \%$, respectively for the presence of > $25 \mathrm{PMNL} / \mathrm{LPF}, 45 \%, 60 \%$, $69.2 \%$ and $35.2 \%$, respectively for the presence of 10-25 PMNLLPF, while the presence of $<10$ PMNL / LPF had sensitivity, specificity, PPV, and NPV of 5 $\%, 70 \%, 25 \%$ and $26.92 \%$, respectively.

There was good correlation between sputum smear results and culture results. A statistically significant difference was found between smear positive and smear negative cases as regards culture positivity ( $p$ value $=0.01$ ). Sensitivity, specificity and PPV and NPV of positive Gram stained- smear for predicting culture positivity was $80 \%, 70 \%$, $84 \%$ and $63.6 \%$, respectively. The results of Gram smear examination of sputum specimens were the same before and after using the wash technique for specimens except in one case with $H$. influenzae in which the organism appeared only after and not before wash and concentration. For $S$. pneumonae, although the smear positivity was the same before \&after wash, however, detection of the organism was better after washing and concentration of the specimens (figure 2).

AECOPD is defined as an acute sustained worsening of the patient's condition from the stable state and necessitates a change in regular medications in a patient with underlying COPD (Basu et al., 2013). Studies revealed that $50-70 \%$ of AECOPD are due to respiratory infections including bacteria, atypical organisms and respiratory viruses, $10 \%$ are due to environmental pollution; depending on season and geographical placement; and up to $30 \%$ are of unknown etiology (Sapey and Stockley, 2006). This study was conducted to investigate the pattern of bacterial pathogens associated with AECOPD and their antibiotic sensitivity profile and to assess the quantitative culture technique of washed sputum specimens

In the current study, to minimize risk of dealing with contaminants, quantitative culture of washed sputum samples was used for diagnosis of bacterial causes of AECOPD in the studied cases. A PPM had to grow above an arbitrary cutoff point to be considered a potential causative agent of an exacerbation (Miravitles et al., 1999; Allegra et al., 2005). Accordingly, bacterial infections were responsible for $66.7 \%$ of all cases of AECOPD. This was in accordance with other studies done in Egypt where the reported rate for bacterial infections in AECOPD was around 60\% (ElKorashy and ElSherif, 2014; Morsi and Degady 2014). Similar rates were reported from Saudi Arabia (69.8\%) (Alamoudi, 2007), Taiwan (66.4\%) (Lin et al., 2007) and France (59\%) (Roche et al., 2007), while lower rates were reported from India (around 42\%) (Basu et al., 2013; Rakesh et al., 2013; Sharan, 2015) and China (34.3\%) (Ma et al., 2015).

Regarding the different types of isolated bacteria in the current study, Gram-positive organisms represented $80 \%$ of the isolates, while Gram-negative organisms represent the remaining $20 \%$. The most prevalent isolated bacteria were $S$. pneumoniae (43.3\%), followed by MRSA (10\%), $H$. influenzae (6.7\%), and lastly $M$. catarrhalis and Acinobacter spp. (3.3\% for each). The results agree with some studies done in Egypt (Alam El-Din, 2002; EL- Batanoony, 2002; Morsi, 2009) which reported that the most prevalent micro-organism in AECOPD was $S$. pneumoniae followed by $S$. aureus or $H$. influenzae then enteric or nonfermentative Gram-negative bacilli. Some studies also in Saudi Arabia (Alamoudi, 
2007) India (Rakesh et al., 2013) and France (Roche et al., 2007) reported that $S$. pneumoniae, $H$. influenzae or $M$. catarralis were the predominant isolates then come the gram negative bacilli. On the other hand, other studies in Egypt (ElKorashy and ElSherif, 2014; Sobhy et al., 2015), India (Basu et al., 2013) and China (Ma et al., 2015) reported predominance of Gramnegative bacilli with $K$. pneumonia, $P$. aeruginosa and Acinetobacter as the most common isolates followed by $S$. aureus. The difference in the prevalence of isolated bacteria depends largely on the types of patients included with predominance of $S$. pneumoniae, $H$. influenzae and $M$. catarralis in community-based outpatients and Gramnegative bacilli from hospitalized patients. In addition, COPD severity is an important determinant of micro-organism type, with gram-negative bacilli being associated with more severe cases (Ma et al., 2015). In the current study, all the isolates were recovered from outpatients except one MRSA and one Acinetobacter isolate which were recovered from 2 inpatients. The prevalence of $H$. influenzae in this study was lower than that reported worldwide. This was in accordance with other studies in India (Sharan, 2015) where no $H$. influenzae was isolated in that study. The authors explain this finding by the temperature variation as well as abuse of antibiotics.

Because of the time required to establish the significance, identity and susceptibility of bacterial isolates from patients with acute respiratory infections, antimicrobial therapeutic choices are usually empirical. The increasing prevalence of resistant organisms, however, complicates this choice and poses a serious threat to current and future treatment of these infections (Sener et al., 2007). As a consequence of acute exacerbations, patients with COPD receive frequent courses of antimicrobial treatment, which has been associated directly with a higher prevalence of resistant microorganisms (Pérez-Trallero et al., 2011). Several studies have reported resistance of pneumococci to penicillins and several other classes of antimicrobials including macrolides, co-trimoxazole and also fluoroquinolones (Sener et al., 2007). It was reported that S. pneumoniae isolates obtained from AECOPD patients were more resistant to the antimicrobial agents generally used in the treatment of pneumococcal infections than those isolated from patients with pneumonia (PérezTrallero et al., 2011). Other studies reported high rate of methicillin resistance among $S$. aureus isolates (Basu et al., 2013) and betalactamase enzyme production by $H$. influenzae and $M$. catarrhalis isolates (Doern et al., 2005). In the current study, penicillin and erythromycin were the least effective against pneumococcal isolates (46\% and 69\%, respectively), while ciprofloxacin and vancomycin were the most effective (92\%). All S. aureus isolates were MRSA. Amoxycillin-clavulinic acid was effective against $M$. cataralis but not $H$. influenzae. The only Acinetobacter isolate was resistant to $3^{\text {rd }}$ generation cephalosporins. Ciprofloxacin and ceftriaoxone were effective against most of the bacterial isolates.

The majority of the patients $(93.3 \%)$ in the current study were males. The patients' age was above 40 . Most patients $(36.7 \%)$ were in the age group from 60 to 70 years with age group 50-60 years old having the highest culture positivity $(100 \%)$. They were either smokers or ex-smokers. Similarly, several studies (Basu et al., 203; Ma et al., 2015; Sharan, 2015) reported almost same age distribution of patients with predominance of males. The high prevalence of AECOPD in advance age group could be explained by impairment of 
immunological defence mechanism, associated co-morbid illness, increased duration of seasonal variation and tobacco smoking, while males were affected more than females because they were more involved in smoking. In non-smokers, especially among women, exposure to indoor air pollution is an important factor (Sharan, 2015). In agreement with other studies (Groenewegen and Wouters, 2003; Alamoud, 2007), no statistical significant difference was found in the current study in sputum culture results in AECOPD with age, sex and smoking.

In this study, there was no statistical significant difference between COPD subgroups as regards culture results or the different types of isolated bacteria. This was in disagreement with some studies (Groenewegen and Wouters, 2003) which observed that the proportion of patients with bacterial infection was higher in those with FEV1 $<50 \%$ predicted, however, in agreement with our results, they did not identify a positive relationship between the type of bacteria and lung function. The results in our study could be explained by that $S$. pneumonia represent the majority of isolates and it was reported that in AECOPD, high rate of Enterobactericae and Pseudomonas spp. in are isolated from patients with worse lung function, while high rate of $S$. pneumoniae, $H$. influenze and M. catarrhalis are isolated in sputum cultures of patients with better lung function (Eller et al., 1998; Larsen et al., 2009).

The current study showed that there was no statistical significant difference in blood gases regarding culture results; however, there was a statistical significant difference between hypoxic and non-hypoxic groups regarding different types of isolated bacteria. This was in agreement with other studies (Groenewegen and Wouters, 2003), however, others (Tsimogianni et al., 2009) found that $\mathrm{PaO} 2$ differed significantly between patients with positive and negative sputum cultures. The different isolated microorganisms in these studies may explain the different results.

Table.1 Sputum Culture Results

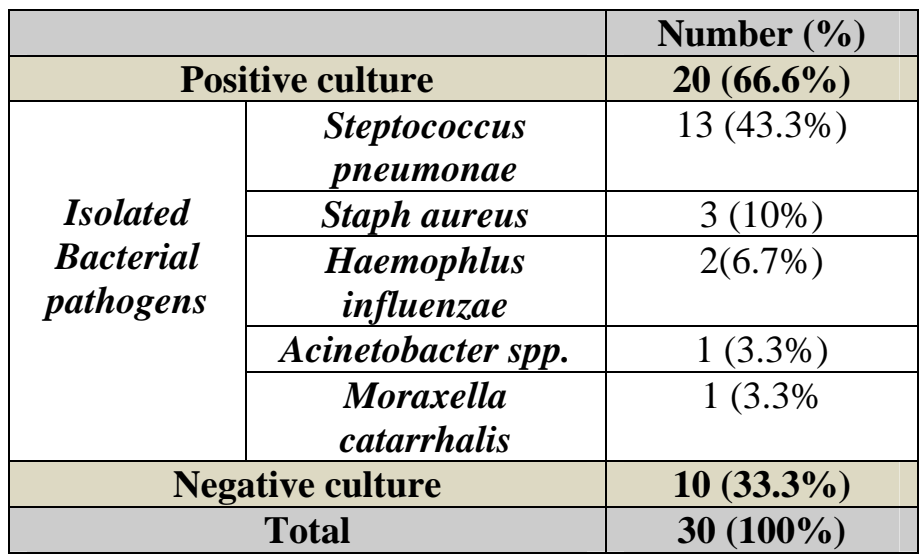


Table.2 Demographic, Clinical and Laboratory Findings of the Patients and their Relation with Culture Results

\begin{tabular}{|c|c|c|c|}
\hline Variables & $\mathbf{N}(\%$ among total patients) & $\begin{array}{c}\text { Culture positive cases } \\
\mathbf{N}(\%)\end{array}$ & $p$ value* \\
\hline $\begin{array}{c}\text { Age groups } \\
40-50 \\
50-60 \\
60-70 \\
>70 \mathrm{yr}\end{array}$ & $\begin{array}{c}8(26.7 \%) \\
8(26.7 \%) \\
11(36.7 \%) \\
3(10 \%)\end{array}$ & $\begin{array}{c}3(38 \%) \\
8(100 \%) \\
7(63.6 \%) \\
2(66.7 \%)\end{array}$ & 0.07 \\
\hline $\begin{array}{c}\text { Smoking } \\
\text { Ex-smoker } \\
\text { Smoker }\end{array}$ & $\begin{array}{c}7(23.3 \%) \\
23(76.6 \%)\end{array}$ & $\begin{array}{c}5(71.4 \%) \\
15(65.2 \%)\end{array}$ & 1.00 \\
\hline $\begin{array}{c}\text { BMI } \\
\quad<22 \\
>22 \mathrm{~kg} / \mathrm{m}^{2}\end{array}$ & $\begin{array}{l}14(46.7 \%) \\
16(53.3 \%)\end{array}$ & $\begin{array}{c}11(78.6 \%) \\
9(56.3 \%)\end{array}$ & 0.26 \\
\hline $\begin{array}{c}\text { Residence } \\
\text { Rural } \\
\text { Urban }\end{array}$ & $\begin{array}{c}5(16.7 \%) \\
25(83.3 \%)\end{array}$ & $\begin{array}{c}4(80 \%) \\
16(64 \%)\end{array}$ & 0.64 \\
\hline $\begin{array}{c}\text { COPD subgroups } \\
\text { Mild } \\
\text { Moderate } \\
\text { Sever } \\
\text { Very severe } \\
\end{array}$ & $\begin{array}{c}2(6.7 \%) \\
10(33.3 \%) \\
8(26.7 \%) \\
10(33.3 \%) \\
\end{array}$ & $\begin{array}{c}2(100 \%) \\
7(70 \%) \\
4(50 \%) \\
7(70 \%) \\
\end{array}$ & 0.55 \\
\hline $\begin{array}{c}\text { Hypoxia } \\
\text { With } \\
\text { Without }\end{array}$ & $\begin{array}{c}3(10 \%) \\
27(90 \%)\end{array}$ & $\begin{array}{l}3(100 \%) \\
17(63 \%)\end{array}$ & 0.53 \\
\hline $\begin{array}{c}\text { Hypoxaemia } \\
\text { With } \\
\text { Without }\end{array}$ & $\begin{array}{l}21(70 \%) \\
9(30 \%)\end{array}$ & $\begin{array}{c}14(66.7 \%) \\
6(66.7 \%)\end{array}$ & 0.66 \\
\hline $\begin{array}{c}\text { Leucocytosis } \\
\text { Yes } \\
\text { No } \\
\end{array}$ & $\begin{array}{r}8(26.7 \%) \\
22(73.3 \%) \\
\end{array}$ & $\begin{array}{c}8(100 \%) \\
12(54.4 \%)\end{array}$ & $0.02 *$ \\
\hline $\begin{array}{l}\text { CRP } \\
\text { Positive } \\
\text { Negative }\end{array}$ & $\begin{array}{c}26(86.7 \%) \\
4(13.3 \%)\end{array}$ & $\begin{array}{c}19(73.1 \%) \\
1(25 \%)\end{array}$ & 0.095 \\
\hline
\end{tabular}

$* p$ value $\leq 0.05$ is considered statistically significant 
Table.3 Distribution of the Isolated Bacterial Pathogens among the Different COPD Subgroups

\begin{tabular}{|c|c|c|c|c|c|}
\hline & \multicolumn{4}{|c|}{ COPD subgroups } & \multirow{3}{*}{$p$ value* } \\
\hline & $\begin{array}{c}\text { Mild } \\
(\mathrm{N}=2)\end{array}$ & $\begin{array}{c}\text { Moderate } \\
(\mathrm{N}=7)\end{array}$ & $\begin{array}{l}\text { Severe } \\
(\mathrm{N}=4)\end{array}$ & $\begin{array}{c}\text { Very Severe } \\
(\mathbf{N}=7)\end{array}$ & \\
\hline & $\mathbf{N}(\%)^{* *}$ & $\mathbf{N}(\%)^{* *}$ & $\mathbf{N}(\%)^{* *}$ & $\mathbf{N}(\%)^{* *}$ & \\
\hline S. pneumoniae & $2(100 \%)$ & $4(57.1 \%)$ & $4(100 \%)$ & $3(42.9 \%)$ & \multirow{5}{*}{0.53} \\
\hline MRSA & $0(0 \%)$ & $2(28.6 \%)$ & $0(0 \%)$ & $1(14.3 \%)$ & \\
\hline H. influenzae & $0(0 \%)$ & $0(0 \%)$ & $0(0 \%)$ & $2(28.6 \%)$ & \\
\hline M. catarrhalis & $0(0 \%)$ & $1(14.3 \%)$ & $0(0 \%)$ & $0(0 \%)$ & \\
\hline Acinetobacter & $0(0 \%)$ & $0(0 \%)$ & $0(0 \%)$ & $1(14.3 \%)$ & \\
\hline
\end{tabular}

${ }^{*} p$ value $\leq 0.05$ is considered statistically significant

** (\%) within COPD subgroups

Table.4 Correlation between Culture Result and Clinical and Laboratory Data

\begin{tabular}{|c|c|c|}
\hline \multirow{2}{*}{ Smoking } & Culture result \\
\hline \multirow{2}{*}{ BMI groups } & Correlation Coefficient & -0.05 \\
\cline { 2 - 3 } & $p$ value & 0.77 \\
\hline \multirow{2}{*}{ FEV1\% group } & Correlation Coefficient & -0.23 \\
\cline { 2 - 3 } & $p$ value & 0.20 \\
\hline \multirow{2}{*}{ SO2 } & Correlation Coefficient & -0.08 \\
\cline { 2 - 3 } & $p$ value & 0.65 \\
\hline \multirow{2}{*}{ PaO2 } & Correlation Coefficient & 0.025 \\
\cline { 2 - 3 } & $p$ value & 0.89 \\
\hline \multirow{2}{*}{ PaCO2 } & Correlation Coefficient & -0.09 \\
\cline { 2 - 3 } & $p$ value & 0.62 \\
\hline \multirow{2}{*}{ Hct } & Correlation Coefficient & -0.16 \\
\cline { 2 - 3 } & $p$ value & 0.37 \\
\hline \multirow{2}{*}{ PLT } & Correlation Coefficient & -0.48 \\
\cline { 2 - 3 } & $p$ value & $0.006 *$ \\
\hline \multirow{2}{*}{ TLC } & Correlation Coefficient & 0.26 \\
\cline { 2 - 3 } & $p$ value & 0.16 \\
\hline \multirow{2}{*}{ CRP } & Correlation Coefficient & 0.44 \\
\cline { 2 - 3 } & $p$ value & $0.013 *$ \\
\hline \multirow{2}{*}{$*$ value $\leq 0.05$ is considered statistically significant } & 0.34 \\
\hline
\end{tabular}

Figure.1 Quantitative Sputum Culture using of 1/10 (a) and 1/100 (b) Dilutions of Washed Sputum Specimens
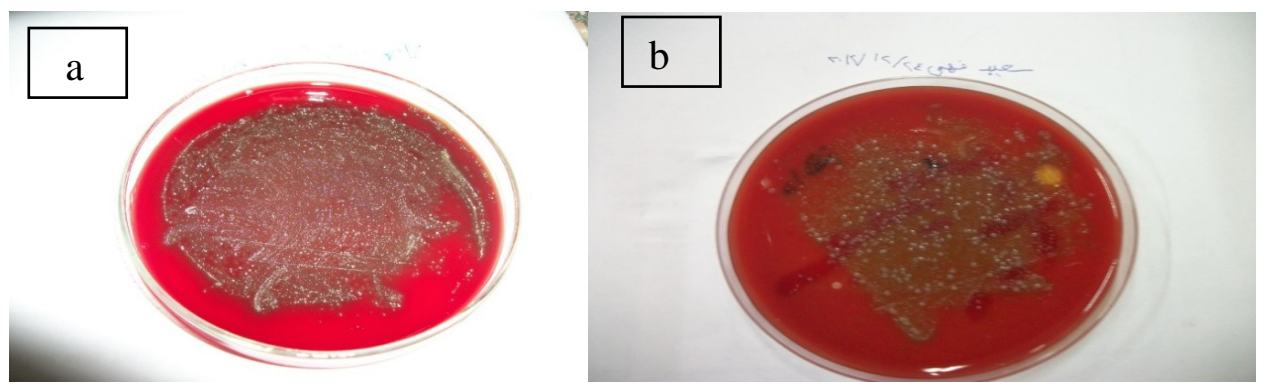
Table.5 Bacteriological Characteristics of Sputum Specimens of Patients

\begin{tabular}{|c|c|c|c|c|}
\hline Variables & $\begin{array}{l}\mathrm{N}(\% \text { among } \\
\text { total patients })\end{array}$ & $\begin{array}{c}\text { Culture } \\
\text { positive cases } \\
\mathbf{N}(\%)\end{array}$ & 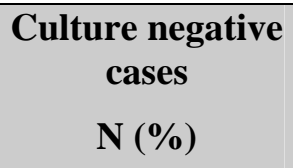 & $p$ value* \\
\hline $\begin{array}{c}\text { Sputum charcter: } \\
\bullet \quad \text { Mucoid } \\
\bullet \quad \text { Mucopurulent } \\
\bullet \quad \text { Purulent } \\
\end{array}$ & $\begin{array}{l}4(13.3 \%) \\
13(43.3 \%) \\
13(43.3 \%) \\
\end{array}$ & $\begin{array}{c}1(25 \%) \\
7(53.8 \%) \\
12(92.3 \%) \\
\end{array}$ & $\begin{array}{c}3(75 \%) \\
6(46.2 \%) \\
1(7.7 \%) \\
\end{array}$ & 0.02 \\
\hline $\begin{array}{c}\text { Leukocytes in sputum : } \\
\qquad \quad>25 \text { leukocyte / LPF } \\
\bullet \quad 10-25 \text { leukocyte / LPF } \\
\text { - } \quad>10 \text { leukocyte / LPF } \\
\end{array}$ & $\begin{array}{c}13(43.3 \%) \\
13(43.3 \%) \\
4(13.3 \%)\end{array}$ & $\begin{array}{c}10(76.9 \%) \\
9(69.2 \%) \\
1(25 \%)\end{array}$ & $\begin{array}{c}3(23.1 \%) \\
4(30.8 \%) \\
3(75 \%) \\
\end{array}$ & $\begin{array}{c}0.4 \\
1.00 \\
0.9 \\
\end{array}$ \\
\hline $\begin{array}{c}\text { Gram-stained Smear } \\
\bullet \quad \text { Positive } \\
\bullet \quad \text { Negative }\end{array}$ & $\begin{array}{l}19(63.3 \%) \\
11(36.7 \%)\end{array}$ & $\begin{array}{c}16(84.2 \%) \\
4(36.4 \%)\end{array}$ & $\begin{array}{l}3(15.8 \%) \\
7(63.7 \%)\end{array}$ & 0.01 \\
\hline
\end{tabular}

${ }^{*} p$ value $\leq 0.05$ is considered statistically significant

Figure.2 A Sputum Specimen Showing S. pneumoniae before (a) and after Wash (b) (x1000). S. pneumoniae Appear more Obvious after Wash (b)

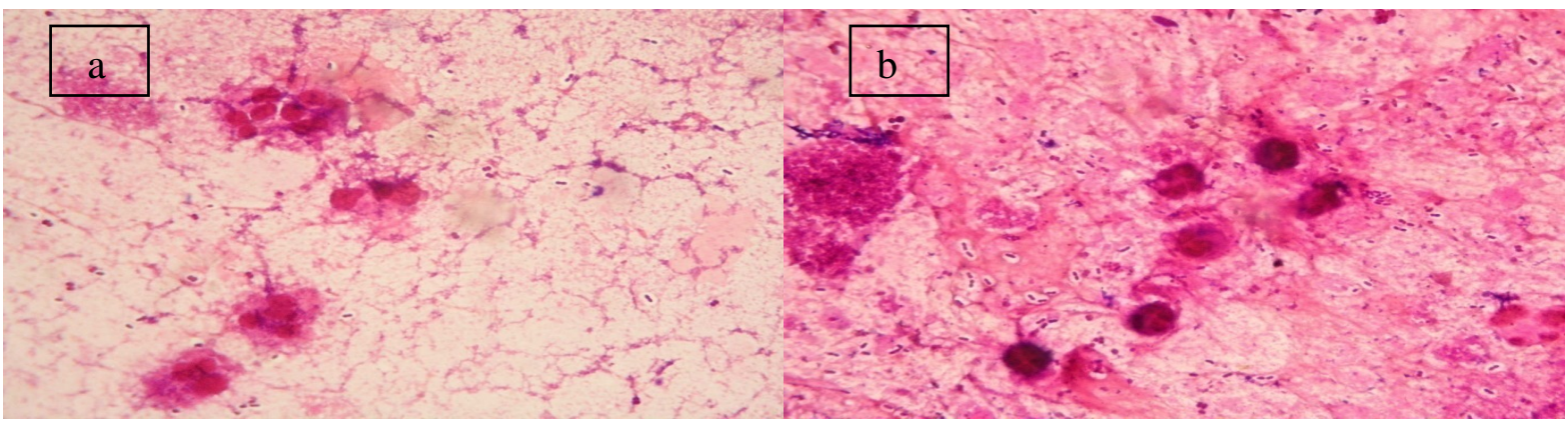

Several studies (Sin and Man, 2003; Roche et al., 2007) reported that WBCs were elevated in a directly proportional manner with severity of AECOPD due to the systemic inflammatory burden. However, others (Bircan et al., 2006) found a normal WBC count and markedly elevated CRP level in AECOPD. In the current study, there was a statistical significant difference between high TLC group and normal TLC group regarding culture results but not as regards type of isolated bacteria. However, high TLC count had low sensitivity (40\%) but excellent specificity (100\%) for predicting positivity of the culture. This was in agreement with other studies (ElKorashy and ElSherif, 2014) which reported that high TLC count was not predominant in most cases of AECOPD.

CRP is an acute phase protein which rises in response to inflammation. Recently, there is evidence that CRP is not just a marker of COPD, but contributes to pathogenesis and increases during AECOPD (Morsi and Degady 2014). In the current work, there 
was no statistically significant difference between patients with positive or negative CRP as regards culture positivity. However, positive CRP had $95 \%$ sensitivity but $30 \%$ specificity for prediction of culture positivity. Other studies also found that CRP was sensitive marker for airway inflammation and systemic inflammation which increase during AECOPD (Sethi and Murphy, 2008; Morsi and Degady 2014). It was reported that CRP can increase during AECOPD triggered by viral infections (Rohde et al., 2008) and this can explain the low specificity in this study.

The presence of green purulent as opposed to white mucoid sputum was reported to be one of the best and easiest methods of predicting a high bacterial load in respiratory tract secretions and the need for antibiotic therapy (Stockely et al., 2000). It was suggested that the presence of bacteria as commensals in the airway could be separated from those causing an infection by increased neutrophil recruitment to the airways in the later a result of activation of host defenses. This neutrophil influx would be associated with a change in secretions from mucoid to purulent (because the myeloperoxidase from the neutrophils is green), and the process would reverse after antibiotic therapy that reduced or eliminated the bacterial load, thereby leading to resolution of the secondary host response. (Stockley, 1998).

In this study, among positive culture, $60 \%$ of cases had purulent sputum, 35\% had mucopurulent sputum and 5\% had mucoid sputum with a statistical significant difference among different physical characters of sputum as regards culture results. Sensitivity, specificity, PPV and NPV of purulent and mucopurulent sputum for prediction of culture positivity were $95 \%, 30 \%, 73 \%$ and $75 \%$, respectively. This was in accordance with other studies (Tsimogianni et al., 2009) which confirmed the predictive role of sputum purulence for positive sputum bacteriology. Other studies have demonstrated the high accuracy of using mucoid sputum to rule out bacterial infection (Stockely et al., 2000).

The main obstacle for using sputum as a diagnostic tool for LRTI is obtaining a good quality (Ziyade and Yagci, 2010). The sputum Gram stain, a standard procedure in clinical microbiology, is used for assessment of specimen quality, and for preliminary, rapid diagnostic information (Campbell and Forbes, 2011). In our study, $88 \%$ of the samples (30 out 34 specimens) were subjected for culture with fewer than 10 SEC /LPF, however, only 38.2\% had > 25 PMNL/LPF. Other studies reported comparable rates for adequate specimens (25-55\%) (Ziyade and Yagci, 2010).

Development of specific immune responses to the infecting bacterial strains, and the association of neutrophilic inflammation with bacterial exacerbations supports the bacterial causation of a proportion of exacerbations (Sethi et al., 2006). In the current study, the presence of PMNLs in Gram stained sputum smears had sensitivity, specificity, PPV, and NPV of 50\%, $70 \%$, $76.9 \%$ and $41 \%$, respectively for the presence of > $25 \mathrm{PMNL} / \mathrm{LPF}$, while the presence of $<10$ PMNL / LPF had sensitivity, specificity, PPV, and NPV of 5 $\%, 70 \%, 25 \%$ and $26.92 \%$, respectively.

The value of the sputum Gram stain for preliminary diagnosis of respiratory disease is well established. In addition, recommendations have been made that sputum culture results be correlated with direct Gram stain results in order to provide more clinically relevant information in light of the limitations of culture (Campbell and 
Forbes, 2011). In this study, there was a good correlation between sputum smear results and culture results. Sensitivity, specificity and PPV and NPV of positive Gram stained- smear for predicting culture positivity was $80 \%, 70 \%, 84 \%$ and $63.6 \%$, respectively. Comparable results were reported in some studies (Parry et al., 2000; Ziyade and Yagci, 2010), while others (Ewing et al., 2002) reported that Gram stain had a low diagnostic yield in community-acquired LRTIs. In the current study, the wash was found to facilitate the detection of $H$. influenzae and $S$. pneumoniae in gram-stained smears. Similar findings were reported in other studies (Ziyade and Yagci, 2010).

In this study, it was shown that using quantitative culture of sputum did not increase the positivity of the culture technique in comparison to the traditional direct inoculation method since all bacterial isolates were detected by both the direct and quantitative methods, however, the quantitative technique helps to exclude 3 false positive cases detected by the direct method. Exclusion of false positive results may help to avoid unnecessary treatment for colonized patients. These results were in disagreement with other studies (Akan et al., 2006; Ziyade and Yagci, 2010) which showed that quantitative culture of sputum samples significantly increased overall culture positivity. Although quantitative culture of washed sputum specimens has been shown to increase either the sensitivity or the specificity of the culture technique for diagnosis of LRTIs, however, the technique is somewhat tedious and time consuming. For routine use of this technique at clinical microbiology laboratories, cost effective analysis should be performed according to the individual needs of the health care facilities (Akan et al., 2006).

In conclusion, Bacterial infections play a major role in AECOPD. S. pneumonia is responsible for the majority of AECOPD in outpatient settings. Ciprofloxacin followed by ceftrioxone can be used for empirical treatment of these infections. Both TLC and CRP are not good predictors of bacterial infections due to lack of either good sensitivity or specificity. Direct Gramstained smear of sputum specimens has a good correlation with culture results. Although quantitative culture can help to avoid unnecessary treatment of colonized patients; however, further studies are required to assess its cost-effective value.

Limitations of this study include the small number of patients and the small number of inpatients included, in addition to the lack of follow-up data of the patients.

\section{References}

Akan O., Ozyilmaz E., Ahmed K., Uysal S., Gulha M. (2006). Quantitative sputum culture versus direct sputum culture. 6th European Congress of Clinical Microbiology and Infectious Diseases. Nice, France, 2006. Abstract number: p986

Alam El-Din AK. (2002). A Bacteriological study in acute exacerbation of chronic obstructive pulmonary diseases patients. Master thesis in chest diseases, Cairo University, Egypt.

Alamoudi OS (2007). Bacterial infection and risk factors in outpatients with acute exacerbation of chronic obstructive pulmonary disease. Respirology; 12(2): 283-287.

Allegra L, Blasi F, Diano P, Cosentini R, Tarsia P, Confalonieri M, Dimakou K, Valenti V. (2005). Sputum color as a marker of acute bacterial exacerbations of chronic obstructive pulmonary disease. Resp Med; 99: 35742- 747. 
American Thoracic Society (1995). Standards for the diagnosis and care of patients with chronic obstructive pulmonary disease. Am J Respir Crit Care Med; 152 : S77-121.

Anthonisen NR, Manfreda J, Warren CP, Hershfield ES, Harding GK, Nelson NA. (1987). Antibiotic therapy in exacerbations of chronic obstructive pulmonary disease. Ann Intern Med; 106 (2): 196-204.

Baron EJ, Thomson JR (2012). Specimen Collection, Transport and processing: Bacteriology. In Versalovic J, Carroll KC, Funke G, Jorgensen JH, Landry ML, Warnock DW, editors. (ed), Manual of clinical microbiology, 10th ed. ASM Press, Washington, DC, PP: 228-271.

Bartlett JG, Finegold SM. (1978). Bacteriology of expectorated sputum with quantitative culture and wash technique compared to transtracheal aspirates. Am Rev Respir Dis;117 (6): 1019-1027.

Basu S, Mukherjee S, Samanta A (2013). Epidemiological study of bacterial microbiology in AECOPD patients of Kolkata, India. Asian Journal of Pharmaceutical and Clinical Research 6 (1), 112-116.

Bircan A, Kaya O, Gok?rmak M, Ozturk O, Sahin U, Akkaya A. (2006). C-reactive protein, leukocyte count and ESR in the assessment of severity of communityacquired pneumonia. Tubercl Toraks; 54 (1): 22-29.

Buenviaje MB (1989). Quantitative Sputum Culture and Gram Stain: Pulmonary Infection vs. Colonization. Phil J Microbiol Infect Dis; 18(1):28-35

Campbell S, Forbes BA. (2011). The Clinical Microbiology Laboratory in the Diagnosis of Lower Respiratory Tract Infections. J Clin Microbiol; 49(9): S30S33.
Cao LD, Ishiwada N, Takeda N, Nigo Y, Aizawa J, Kuroki H, Kohno Y. (2004). Value of washed sputum Gram stain smear and culture for management of lower respiratory tract infections in children. J Infect Chemother; 10 (1): 3136.

Clinical and Laboratory Standard Institute (CLSI) (2013). Performance Standards for Antimicrobial Susceptibility Testing; Twenty-Third Informational Supplement. CLSI document M 100- S 23. CLSI, Wayne, Pennsylvania, USA, 2013.

Doern GV, Richter SS, Miller A, Miller N, Rice C, Heilman K, Beekmann S. (2005). Antimicrobial resistance among Streptococcus pneumonia in the United States: Have we begun to turn the corner on resistance to certain antimicrobial classes. Clin Inf Dis; 41 (2): 139-148.

EL- Batanoony A. (2002). Yield of sputum in exacerbations ofchronic obstructive pulmonary disease. Master thesis in chest diseases, Cairo University, Egypt.

ElKorashy RIM, El-Sherif RH (2014). Gram negative organisms as a cause of acute exacerbation of COPD. Egyptian Journal of Chest Diseases and Tuberculosis; 63(2): 345-349

Eller J, Ede A, Schaberg T, Niederman MS, Mauch H, Lode H. (1998). Infective exacerbations of chronic bronchitis: relation between bacteriologic etiology and lung function. Chest; 113 (6): 1542-1548.

Ewig S, Schlochtermeter M, Göke N, Niederman MS. (2002). Applying sputum as a diagnostic tool in pneumonia. Limited yield, minimal impact on treatment decisions. Chest; 121:1486-1492. 16.

Geckler RW, GremillionDH, Mcallister CK, Ellenbogen C. (1977). Microscopic and Bacteriological Comparison of Paired 
Sputa and Transtracheal Aspirates. J Clin Microbiol; 6(4): p. 396-399.

Global Initiative for Chronic Obstructive Lung Disease (GOLD) (2010). Global Strategy for the Diagnosis, Management and Prevention of Chronic Obstructive Lung Disease (Updated 2010).

Groenewegen KH, Wouters EF. (2003). Bacterial infections in patients requiring admission for an acute exacerbation of COPD; a 1-year prospective study. Respir Med; 97 (7) : 770-777.

Larsen MV, Janner JH, Nielsen SD, FriisMøller A, Ringbaek T, Lange P. (2009). Bacteriology in acute exacerbation of chronic obstructive

pulmonary disease in patients admitted to hospital. Scand J Infect Dis.; 41(1):26-32.

Lin SH, Kuo PH., Hsueh PR, Yang PC, Kuo SH (2007). Sputum bacteriology in hospitalized patients with acute exacerbation of chronic obstructive pulmonary disease in Taiwan with an emphasis on Klebsiella pneumoniae and Pseudomonas aeruginosa, Respirology 12(1): 81-87..

Ma X, Cui J, Wang J, Chang Y, Fang Q, Bai C, Zhou $\mathrm{X}$, Zhou H, Feng H, Wang Y, Zhao W, Wen Z, Wang P, Liu Y, Yu L, Li C, Chen L. (2015). Multicentre investigation of pathogenic bacteria and antibiotic resistance genes i $\mathrm{n}$ Chinese patients with acuteexacerbatio $\mathrm{n}$ of chronic obstructive pulmonary disea se. J Int Med Res. 2015 Oct;43(5):699710.

Miravitles M, Espinosa C, Fernandez-Laso E, Martos JA, Maldonado JA, Gallego M. (1999). Relationship between bacterial flora in sputum and functional impairment in patients with acute exacerbations of COPD. Chest; 116 (1) : 40-6.

Morris JF, Koski A, Johnson LC. (1971). Spirometric Standards for Healthy Non-
Smoking Adults. Am Rev Respir Dis; 103: 57-67.

Morsi A (2009). Pathogens causing lower respiratory tract infections. Master thesis in chest diseases, Ain Shams University , Egypt.

Morsi TS, Degady AA. (2014). Value of copeptin and $\mathrm{C}$-reactive protein in acute exacerbation of chronic obstructive pulmonary disease. Egyptian Journal of Chest Diseases and Tuberculosis; 63: 853-859.

National Institute for Health and Care Excellence (NICE) (2010). Chronic Obstructive Pulmonary Disease in Over 16s: diagnosis and Management.

Parry CM, White RR, Ridgeway ER, Corkill JE, Smith GW. (2000). The reproducibility of sputum Gram film interpretation. J Infect; 41:55-60.

Pérez-Trallero E, Marimón JM, Larruskain J, Alonso M, Ercibengoa M. (2011). Antimicrobial susceptibilities and seroty pes of Streptococcus pneumoniae isolate $\mathrm{s}$ from elderly patients withpneumonia a nd acute exacerbation of chronic obstruc tive pulmonary disease. Antimicrob Agents Chemother. ; 55(6):2729-34.

Rakesh G, KasturiT., Yuvarajan S. (2013). Bacterial agents causing acute exacerbations in Chronic Obstructive Pulmonary Disease (COPD) patients, their antibiograms to Extended Spectrum BetaLactamases (ESBL) production in a tertiary care hospital, India. Int.J.Curr.Microbiol.App.Sci; 2(11): 273-282.

Roche N, Kouassi B, Rabbat A, Mounedji A, Lorut C, Huchon G. (2007). Yield of sputum microbiological examination in patients hospitalized for exacerbations of chronic obstructive pulmonary disease with purulent sputum. Respiration.; 74(1):19-25.

Rodriguez-Roisin R. (2000). Toward a consensus definition for COPD 
Exacerbations. Chest; 117(5 Suppl 2): 398S-401S.

Rohde G, Borg I, Wiethege A, Kauth M, Jerzinowski S, An Duong Dinh T, Bauer TT, Bufe A, SchultzeWerninghaus G. (2008). Inflammatory response in acute viral exacerbations of COPD. Infection; 36 (5): 427-433.

Sapey E, Stockley RA. (2006). COPD exacerbations. 2: Aetiology. Thorax; 61(3): 250-258.

Sener B, Tunçkanat F, Ulusoy S, Tünger A, Söyletir G, Mülazimoğlu L, Gürler N, Oksüz L, Köksal I, Aydin K, Yalçin AN, Oğünç D, Acar A, Sievers J. (2007). A survey of antibiotic resistance in Streptococcus pneumoniae and Haemophilus influenzae in Turkey, 2004 2005. J Antimicrob Chemother.; 60 (3):587-93.

Sethi S, Evans N, Grant BJ, Murphy TF. (2002): New strains of bacteria and exacerbations of chronic obstructive pulmonary disease. $\mathrm{N}$ Engl $\mathrm{J}$ Med; 347(7): 465-471.

Sethi, S, Maloney J, Grove L, Wrona C, Berenson CS. (2006): Airway inflammation and bronchial bacterial colonization in chronic obstructive pulmonary disease. Am J Respir Crit Care Med; 173(9):991-8.

Sethi S, Murphy TF. (2008): Infection in the pathogenesis and course of chronic obstructive pulmonary disease. N Engl J Med; 359 (22): 2355-65.

Sharan H. (2015): Aerobic Bacteriological Study of Acute Exacerbations of Chronic Obstructive Pulmonary Disea se. J Clin Diagn Res.; 9(8):DC10-2.

Sin DD, Man SF. (2003): Why are patients with chronic obstructive pulmonary disease at increased risk of cardiovascular diseases, Circulation 107(11):1514-1519.

Sobhy KE, Abd El-Hafeez AM, Shoukry FA, Refaai ES. (2015). Pattern of sputum bacteriology in acute exacerbations of chronic obstructive pulmonary disease. Egypt J Bronchol (2015); 9 (2): 170-177.

Stockley R A. (1998): Lung infections 1: Role of bacteria in the pathogenesis and progression of acute and chronic lung infection. Thorax. (53): 58-62.

Stockley RA, O'Brien C, Pye A, Hill SL. (2000): Relationship of Sputum Color to Nature and Outpatient Management of Acute Exacerbations of COPD. Chest; 117(6): 1638-45.

Tsimogianni AM, Papiris SA, Kanavaki S, Stathopoulos GT, Sotiropoulou C, Manali ED, Michalopoulou P, Roussos C, Kotanidou A. (2009). Predictors of positive sputum cultures in exacerbations of chronic obstructive pulmonary disease. Respirology; 14(8):1114-20.

Ziyade N, Yagci A. (2010): Improving Sputum Culture Results for Diagnosis Of Lower Respiratory Tract By Saline Washing. Marmara Medical Journal 2010; 23 (1);30-36.

\section{How to cite this article:}

Dalia Saad ElFeky, Heba Mohammed Elmandory, Mohamed Galal and Mohamed Abdel Hakim. 2016. Sputum Bacteriology in Patients with Acute Exacerbation of Chronic Obstructive Pulmonary Disease. Int.J.Curr.Microbiol.App.Sci. 5(1): 289-305 hittp: //dx. doi.org/10.20546/ijcmas.2016.501.028 